\title{
MECANISMOS DEMOGRAFICOS \\ Y OSCILACIONES A LARGO PLAZO \\ DE LA POBLACION EUROPEA (1200-1850) *
}

\author{
VICENTE PEREZ MOREDA y DAVID-SVEN REHER \\ Universidad Complutense
}

\section{Introducción}

Explicar los mecanismos demográficos que intervienen en las oscilaciones a largo plazo de la población es una espinosa tarea, especialmente difícil cuando la calidad de los datos que se manejan no es la más adecuada y si se pretende resumir dicha explicación en unas pocas páginas. Como es sabido, no existe en ningún lugar de Europa, con anterioridad al siglo xvi, información precisa sobre las diferentes variables demográficas, ni siquiera sobre las tendencias de la población total; la anterior a 1750 es escasa y muchas veces dudosa, y sólo empieza a ser adecuada en algunas zonas después de esa fecha. A pesar de todo, el estado actual de los conocimientos sobre las poblaciones del pasado nos permite intentar algún tipo de síntesis arriesgada sobre la naturaleza de los cambios demográficos que Europa ha registrado desde la Baja Edad Media, en la línea del trabajo de Flinn (1981), aunque con un enfoque cronológico y analítico diferente. Para simplificar al máximo nuestro intento, incluiremos sólo aquellas zonas de Europa que cuentan al menos con una información rigurosa mínima sobre la que podamos especular, aunque eso suponga dejar fuera de nuestra observación a casi la mitad del continente. Intentando especificar comportamientos generales, toda la discusión se desarrollará a niveles nacionales o incluso supranacionales, lo que impedirá la consideración de buena parte de la variabilidad regional tan característica de muchas zonas europeas.

Una rápida observación de las tasas de crecimiento demográfico, a lo largo de los siglos considerados, nos permite establecer ya algunas conclusiones generales. El cuadro adjunto muestra esas tasas en cada una de las regiones europeas analizadas, entre 1200 y 1850 . La primera conclusión que se des-

* Una primera versión de este trabajo se presentó en el XX Congreso General de la Unión Internacional para el Estudio Científico de la Población (Florencia, junio de 1985) y se publicó en las Actas de dicho Congreso: IUSSP, International Population Conference, Florencia, 1985, IV: 313-329. 


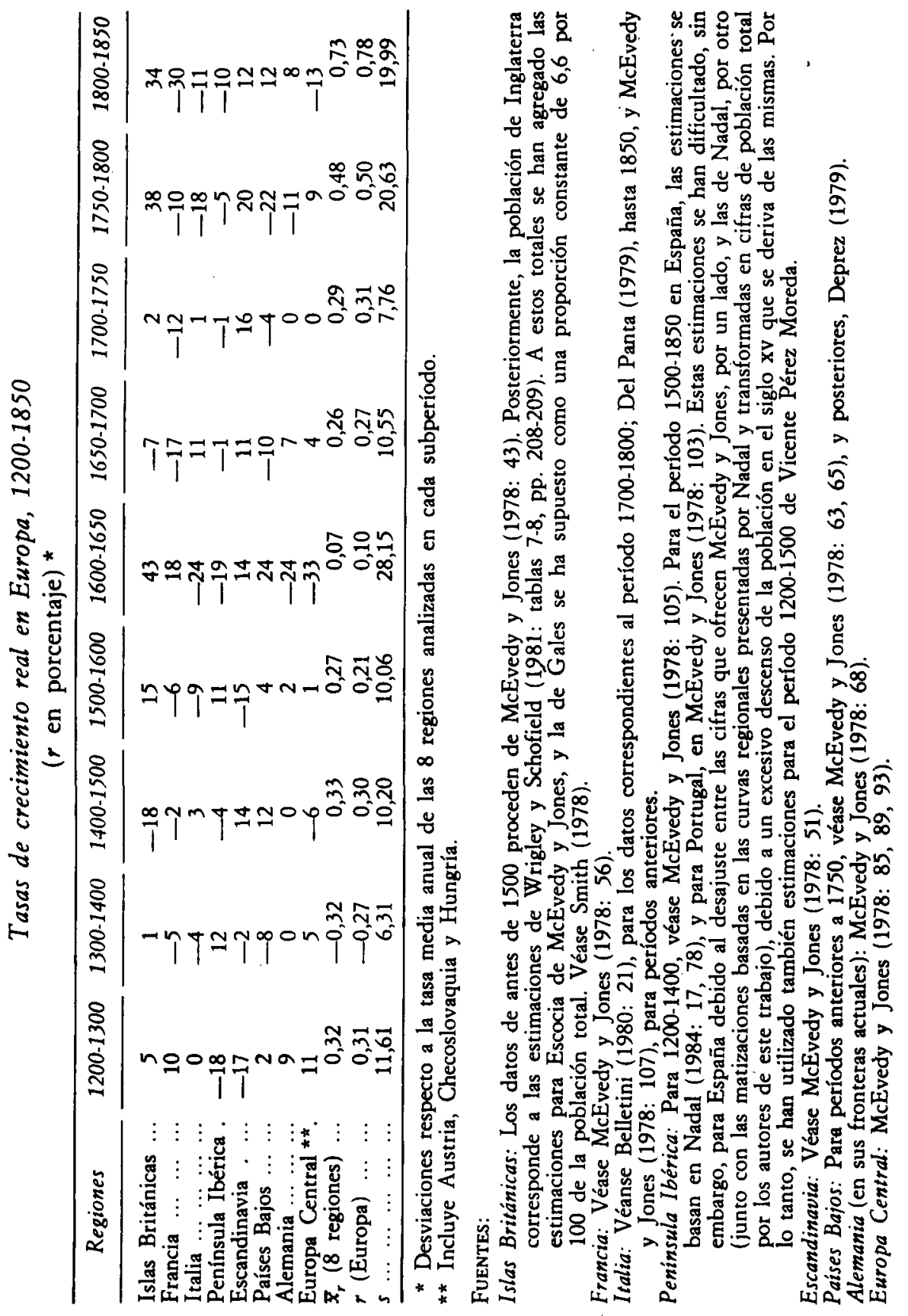


prende de estos datos es que las tasas de crecimiento situadas entre el 0,25 y el 0,35 por 100 anual parece haber sido la norma habitual en la mayor parte del período en cuestión. Las únicas divergencias respecto a esas tasas «normales» quedan relegadas al siglo XIV, a la primera mitad del siglo xviI y al período posterior a 1750 . Otro aspecto llamativo de la información contenida en el cuadro reside en la relativa homogeneidad de las tasas de crecimiento de las diferentes regiones europeas en cada subperíodo, excepto en el que transcurre de 1600 a 1650 y, de nuevo, el posterior a 1750. Todo esto nos sugiere un posible significado clave de esos períodos «atípicos», y nos plantea inevitablemente dos cuestiones fundamentales: ¿A qué se debe esa semejanza general de las fluctuaciones demográficas a largo plazo entre unas regiones y otras? ¿Por qué esa divergencia que se manifiesta entre ellas en ciertas épocas? La discusión de estas cuestiones será el principal objetivo de estas páginas.

\section{La begemonia de la mortalidad}

Intensas, aunque breves, fluctuaciones de la mortalidad parecen haber desempeñado un papel central en la determinación de las oscilaciones a largo plazo de la población europea hasta tiempos relativamente recientes, y sería imprudente negar su importancia. La intensidad y frecuencia de repetidas crisis de mortalidad podían convertir las tasas positivas de crecimiento neto en pérdidas. Con motivo de tales crisis periódicas, otras variables demográficas fueron ajustándose constantemente para afrontar la situación impuesta por las fluctuaciones de la mortalidad. Si Dupâquier (1972; 1979: 381-391) ha podido afirmar que la nupcialidad fue el principal mecanismo autorregulador de las sociedades del Antiguo Régimen, la verdad es que en la mayoría de los ejemplos que él cita la nupcialidad se ve obligada a intervenir para colmar los vacíos originados por una crisis determinada. No se trata de negar, por supuesto ${ }_{6}$ la influencia respectiva de las demás variables demográficas -incluidos los movimientos migratorios- sobre las oscilaciones a largo plazo de la población europea en los primeros siglos de su historia abordados en este estudio, sino de subrayar más bien que su intervención y sus efectos quedaban condicionados por la imperiosa necesidad de compensar las intensas fluctuaciones de la mortalidad.

Según Malthus, había en las sociedades tradicionales un delicado equilibrio entre la presión demográfica y los recursos, y siempre que el crecimiento de la población situaba los niveles de vida por áebajo del nivel mínimo de subsistencia debían entrar en acción ciertos mecanismos para restablecer dicho 
equilibrio. Mientras que las poblaciones «prudentes» (donde el crecimiento demográfico era controlado principalmente a través de la nupcialidad) nunca habrian llegado a ese mínimo de subsistencia, en las demás, la mortalidad, el clásico "freno correctivo", habría tenido que intervenir para restaurar la situación anterior. Las graves crisis de mortalidad de las sociedades del Antiguo Régimen podrían ser, en principio, ejemplos perfectos de este mecanismo; siempre, claro está, que pueda mostrarse una evidente relación con descensos correspondientes en los niveles de vida o, para decirlo lisa y llanamente, con el hambre. Algunos historiadores, como Goubert (1968: 68-82) o Lebrun (1980: 207), seducidos por las fluctuaciones de los precios que habitualmente acompañaban a las crisis de mortalidad, han suscrito este punto de vista, pero un creciente número de especialistas han advertido que la mortalidad pocas veces muestra más que una débil correlación con los precios y algunos de ellos han llegado incluso a sugerir que los fenómenos epidémicos eran en muchas ocasiones causa, y no consecuencia, de las fluctuaciones de la producción y los precios (Dupâquier, 1979: 377-378; R. Lee, 1978: 179186; Wrigley y Schofield, 1981: 313-332, 368-384; Pérez Moreda, 1980: $77-82,373-374 ; 1984)$. Por lo general, la muerte difícilmente puede ser atribuida de forma directa al hambre, y la etiología de las diferentes enfermedades más graves del pasado nos muestra su naturaleza totalmente exógena (caso de la peste), o bien sólo indirectamente relacionada con los niveles de vida (caso del tifus). Por otra parte, existen importantes ejemplos de un declive de los niveles de mortalidad en áreas, como la Francia del siglo xviII, que no manifiestan ningún progreso notable en sus niveles de vida. No se trata aquí de refutar a Malthus, sino de afirmar que, a la hora de condicionar el crecimiento de una población, la mortalidad exógenamente determinada también jugaba a menudo el papel protagonista. No dudamos que esta mortalidad actuase de hecho como un freno correctivo a la población, sino simplemente afirmamos que su actuación, aun teniendo una gran incidencia en el sistema demográfico, no fue más que muy indirectamente producto del mismo.

De esta manera, hasta tiempos recientes el crecimiento de la población estuvo condicionado por unos altos niveles de mortalidad que reflejaban, naturalmente, la intensidad y frecuencia de la mortalidad catastrófica, atribuible por lo común a causas exógenas. Las tasas de crecimiento, que en ausencia de crisis podían alcanzar fácilmente el 0,5 por 100 anual, o más, se mantuvieron de hecho por debajo del 0,3 por 100 durante largos períodos de tiempo, y en especiales circunstancias pudieron caer incluso a niveles apreciablemente inferiores. La peste fue, sin duda, una de las más importantes causas de mortalidad catastrófica; el tifus, la gripe, la viruela, el paludismo, la sífilis, la fiebre amarilla, el cólera y otras enfermedades infecto-contagiosas des- 
empeñaron un papel menos decisivo a corto plazo, aunque algunos especialistas sostienen que el tifus podría haber causado más víctimas, a fin de cuentas, que la misma peste (Del Panta y Livi Bacci, 1977: 416-419). La incidencia de la mortalidad catastrófica disminuyó, sin embargol gradualmente en la mayor parte de Europa en sucesivos momentos entre 1675 y 1800 . Este retroceso, debido en gran medida a la casi definitiva desaparición de la peste entre 1675 y 1721, fue más precoz en la Europa nórdica que en la meridional, pero acabó afectando a todo el continente. Cambios en la naturaleza interna de las enfermedades epidémicas (McNeill, 1976; Appleby, 1980), mejoras en la higiene y en la organización administrativa de defensa de las poblaciones ante el contagio (McKeown, 1978; Biraben, 1975-76; Flinn, 1979; Slack, 1981), cambios climáticos (Galloway, 1984; Biraben, 1975-76) y, por lo que respecta a los niveles generales de mortalidad, una mejor nutrición (McKeown, 1976), han sido las principales causas citadas como responsables de esta «estabilización de la mortalidad» (Flinn, 1974). Cualesquiera que fueran sus orígenes, el declive de la mortalidad epidémica se vio acompañado de tasas de crecimiento más altas y cada vez más heterogéneas, que eran el producto final de la peculiar interacción de las variables características de los diferentes sistemas demográficos existentes en Europa.

Entre 1300 y 1650 la peste ejerció un protagonismo central en el desarrollo de la población europea. A raíz de su llegada a Italia en 1347, en poco más de tres años la Peste Negra afectó a casi todo el continente, matando, quizá, a más de una cuarta parte de sus habitantes. Esta primera irrupción fue seguida por otras, con una periodicidad bastante regular de intervalos de unos 10 a 13 años, durante el resto del siglo xIv y la primera mitad del xv. No resulta muy difícil imaginar los efectos demográficos de esta serie de ataques, pero tal vez un rudimentario ejercicio ayude a describir a grandes rasgos las consecuencias estadísticas de la peste. Si aceptamos una recurrencia de la enfermedad cada 12 años (aunque a niveles locales su presencia pudo ser, de hecho, más habitual), con una pérdida demográfica neta, calculando por lo bajo, de un 20 por 100 en cada ocasión, y suponemos, de forma más bien optimista, una tasa de crecimiento del 1 por 100 anual entre cada epidemia, la pérdida neta de población entre 1348 y 1430 habría sido ligeramente superior al 40 por 100 . Durante este período los niveles generales de mortalidad fueron probablemente bastante más altos que los registrados en Europa en los mil años anteriores. Russell (1979: 50) estimó que la esperanza de vida $\left(e_{o}\right)$ entre los terratenientes ingleses cayó de niveles cercanos o superiores a los 30 años —que se observan antes de 1325 o después de 1425- a niveles inferiores a los 20 años entre 1348 y 1375. En realidad sorprende que las pérdidas demográficas no fueran mayores, lo que probable- 
mente se explica por la decisiva reacción del comportamiento matrimonial, que tendería a maximizar los niveles de fecundidad durante el período. Lo poco que sabemos acerca de la nupcialidad de esos tiempos nos indica que los matrimonios nunca fueron tan precoces como en los momentos inmediatos posteriores a los primeros ataques catastróficos de la peste. La edad de las mujeres en sus primeras nupcias era de 17,6 años en Florencia en 1427, de 19,5 en 1458 y de 20,8 en 1480 ; y en Prato fue de 16,3 años en 1372, 17,6 en 1427 y 21,1 en 1470 (Herlihy y Klapisch-Zuber, 1978). Es probablę que esta reacción de la nupcialidad se produjera en muchos sitios, aunque es difícil conocer su intensidad.

La población alcanzó, sin duda, un punto más bajo, en unas y otras regiones europeas, entre 1380 y 1425 . El balance final fue desastroso, pues ninguna zona importante del continente quedó libre de la epidemia. Tras esta última fecha, se hace evidente una pronunciada recuperación de las tasas de crecimiento. Puede ser que una de las razones de este cambio de tendencia resida en la mejor situación de los campesinos sobrevivientes, a quienes la caída de la población habría facilitado el acceso a la tierra $y$, en consecuencia, al matrimonio; de nuevo en este caso, la peste ostentaría la responsabilidad última de la nueva fase de crecimiento. Aunque la enfermedad siguió siendo un gran obstáculo para el aumento de la población a lo largo del siglo XVI, la intensidad y frecuencia de sus ataques parecen haber disminuido durante buena parte de esta centuria. La información facilitada por Del Panta (1980: 132) es suficientemente elocuente, por lo que a Italia se refiere, y se ve confirmada, al parecer, por pruebas indirectas procedentes de otros países. El siglo xvi fue positivo en todos ellos, aunque las tasas de crecimiento en modo alguno fueran uniformes. El débil crecimiento que registran Italia y Francia acusa los disturbios políticos y militares de la época en ambas zonas. La expansión más rápida de la población inglesa fue debida, con toda seguridad, a unos niveles muy bajos de mortalidad, que mostraron una reacción escasa o nula ante el fuerte declive de los, salarios reales, y que se tradujo en unos valores de $e_{0}$ cercanos a los 40 años (Wrigley y Schofield, 1981: 228236). En el caso de España, sin embargo, parece improbable que la mortalidad llegara a ser verdaderamente baja en algún momento, y una mejor explicación de su crecimiento demográfico tal vez se encuentre en las hasta ahora limitadas pruebas que tenemos acerca de una intensa nupcialidad, estimulada por unas densidades demográficas relativamente bajas, que a su vez sostenían mejor que en otras zonas el salario real y permitían una extensión de los cultivos.

Durante el siglo xvil surgieron contrastes mucho mayores en las tasas de crecimiento, debido otra vez a la distinta cronología y naturaleza de las epi- 
demias, guerras y crisis económicas que afectaron a cada zona. Considerada en toda su extensión, la centuria muestra una tendencia próxima al estancamiento demográfico, aunque algunas regiones presenciaron pérdidas netas de población durante buena parte del período. La primera mitad del siglo fue muy negativa para Italia, España y Alemania, aunque por razones distintas en algún caso. En España, el crecimiento castellano parece atenuarse desde la década de 1570 , debido probablemente a los efectos de una nupcialidad cada vez más restringida, ejemplo que será seguido, con cierto retraso, por las demás regiones peninsulares. En este cambio de la tendencia secular de la nupcialidad debieron de influir tanto un empeoramiento de los niveles de vida y de las posibilidades de acceso a la tierra, como las condiciones mentales y sociales impuestas por el espíritu de la Contrarreforma, que inclinaría a un número creciente de hombres y mujeres hacia el celibato eclesiástico. Aunque es difícil determinar si lo que en realidad hubo fue una reacción ante las dificultades crecientes para la formación de nuevos hogares, o bien un cambio de conducta inspirado por circunstancias culturales, lo cierto es que el fenómeno será frecuentemente mencionado por los escritores reformistas de la época como una de las causas más importantes de la relativa despoblación del país. Una elevación de la edad media en las primeras nupcias femeninas, de 1,5 años aproximadamente, entre la segunda mitad del siglo xVI y la primera del xviI, como es la que se desprende de los escasos datos hasta ahora disponibles, habría afectado notablemente a las tasas de crecimiento, sobre todo si se vio acompañada de un aumento paralelo del celibato definitivo (Bideau, 1984: 1142-1143). Estos sombríos presagios de un estancamientọ de la población dieron paso al desastre demográfico ocasionado por las grandes epidemias de peste de 1596-1602 — tal vez la más importante de toda la centuria - y de 1647-54, así como por otra serie de epidemias de tifus, entre las que destacan las de 1606 y 1630-31 (Pérez Moreda, 1980: 245-326). El declive demográfico se presentó asociado a una importante recesión económica en las zonas urbanas, y todo ello condujo a una gran redistribución de la población española en favor de muchas regiones periféricas y de Madrid. La mayor parte de las zonas afectadas por esta situación empezaron a reaccionar frente a ella a lo largo de la segunda mitad del siglo, aunque sus consecuencias sociales, demográficas y económicas iban a perdurar todavía durante algún tiempo (Nadal, 1984: 73-85). En gran medida, la experiencia italiana comparte un tipo de crisis similar, donde el descenso de los niveles de vida, posibles retrocesos de la nupcialidad, así como una serie de violentas incursiones de la peste y el tifus, son factores que contribuyen todos juntos al declive de la población (Livi Bacci, 1978: 39-58). En el caso de Alemania, la escasa información demográfica dificulta la tarea de estable- 
cer hipótesis fiables, aunque sería arriesgado no reconocer que la guerra de los Treinta Años y las epidemias relacionadas con ella ocasionaron un gran número de pérdidas.

El signo negativo de los saldos migratorios probablemente contribuyó también de forma importante a las tasas de crecimiento modestas, y a veces negativas, que se observan en este período. Las corrientes migratorias dentro del continente, casi las únicas que se pueden tomar en consideración con anterioridad a la colonización del Nuevo Mundo, habian tenido hasta entonces escasos efectos demográficos a escala regional, y nulos -obviamente- a escala continental. Pero a partir de 1500 las migraciones adquirieron una considerable importancia para el crecimiento demográfico, a lo que contribuyó poderosamente el inicio de los flujos migratorios intercontinentales. En Espa$\tilde{n} a$, por ejemplo, el éxodo definitivo ocasionado entre 1500 y 1650 por la emigración a América, las guerras europeas y la expulsión de los moriscos, habría afectado, según un cálculo prudente, a algo más de un millón de personas: una pérdida sólo parcialmente compensada por la entrada de unos 200.000 inmigrantes franceses a los territorios de la Corona de Aragón durante el mismo período (Nadal, 1984: 43-72). También Inglaterra sufrió importantes pérdidas emigratorias durante el siglo xvir (Wrigley y Schofield, 1981: 528-529). Hay que tener en cuenta, sin embargo, que - si exceptuamos la expulsión de los moriscos- los restantes tipos de emigración consistieron en flujos graduales cuyos efectos sobre las tasas de crecimiento neto de la población serían limitados, a diferencia de lo que ocurriría a raíz de continuas reducciones demográficas en el caso de grandes epidemias recurrentes, cuyas repercusiones sobre la estructura de edades y la capacidad reproductora de las poblaciones afectadas habrían de ser más importantes.

En el norte de Europa, el siglo XviI debe ser considerado más bien como un período de estancamiento que de crisis. Las tasas de crecimiento positivo se mantienen allí durante la primera mitad de la centuria y sólo más tarde muestran un retroceso. De nuevo hay que lamentar las limitaciones de la información disponible, que dificultan seriamente la correcta comprensión de las causas de estas fluctuaciones. En el caso de Inglaterra, sin embargo, el panorama ha quedado mucho más claro gracias a la obra de Wrigley y Schofield (1981: 207-248). El descenso de la fecundidad, ya iniciado a finales del siglo XVI, y un recrudecimiento de la mortalidad a partir de 1620 redujeron las tasas de crecimiento entre esta fecha y 1650 , hecho que no se refleja en la tabla adjunta debido a la inclusión en ella de las restantes zonas de las Islas Británicas. Posteriormente, la actuación conjunta de ambas variables eliminó por completo el crecimiento inglés en la segunda mitad del siglo. A partir de 1670 , la fecundidad conoce un rápido incremento como respuesta tardía 
a la mejora de los niveles salariales, aunque una elevación de la mortalidad en la década de 1720 retrasará el definitivo despegue demográfico inglés hasta después de 1740. Si bien ambas variables influyeron conjuntamente en el ritmo de crecimiento durante este período, parece seguro que fue la fecundidad total —estimulada por la nupcialidad más que por posibles cambios registrados en la fecundidad legítima - el factor que ejerció una mayor influencia (Wrigley y Schofield, 1983: 169-170; Wrigley, 1983: 130-133). Es difícil saber si un esquema similar a éste es aplicable a otras zonas. La inflexión del crecimiento que conoció la población francesa fue parecida a la de Inglaterra, aunque la evolución demográfica de ambos países manifiesta profundas divergencias después de 1700. En la pequeña Holanda, densamente poblada y con un alto grado de urbanización, el brillante crecimiento de casi todo el siglo xvil se vio sustituido a finales del período por otra centuria de estancamiento, probablemente originado por el colapso de su prosperidad económica tras la guerra con Francia y sus secuelas de limitadas oportunidades para la nupcialidad, junto a unos niveles más altos de mortalidad típicos de las áreas urbanas de los tiempos modernos (De Vries, 1974: cap. 3).

Más allá de estas diferencias regionales, hacia 1700 un profundo cambio empezó a afectar a toda Europa. A partir de ahora la frecuencia y gravedad de las crisis de mortalidad fueron descendiendo gradualmente en todos los sitios, eliminando así una de las principales explicaciones de las tendencias del tamaño de la población y de los salarios reales (R. Lee, 1973). Una breve alusión a los datos más relevantes nos despeja cualquier duda al respecto. La mortalidad catastrófica alcanzó en Inglaterra, en la segunda mitad del siglo xvili, sólo un tercio del nivel de un siglo anterior; en Francia, un 40 por 100; en Escocia, un 80 por 100; en Italia, un 30 por 100 . Unicamente la España interior parece desviarse de esta tendencia descendente, aunque un agregado nacional de datos podría tal vez modificar esta conclusión (Wrigley y Schofield, 1981: 334; 1974: 293-294; Del Panta, 1980: 153, 196-198; Pérez Moreda, 1980: 126-127). Muchas enfermedades epidémicas siguieron afectando todavía a toda Europa, pero su incidencia global en las tasas de crecimiento había empezado a descender rápidamente. En esta nueva situación, otros mecanismos, que hasta ahora sólo habrían ejercido, al parecer, un relativo protagonismo en la determinación de las tasas de crecimiento, pasaron a asumir un control incontestable de las mismas dentro de los sistemas demográficos que se mirestran cada vez más característicos de la experiencia europea. Por ello, antes de proseguir, parece conveniente explorar algunas de las implicaciones demográficas de dichos sistemas. 


\section{Sistemas demográficos y fluctuaciones de la población}

En diferentes momentos de la historia europea podemos observar sistemas demográficos claramente distintos que coexisten en el continente. Algunas regiones conocen un régimen demográfico caracterizado por una elevada fecundidad y una mortalidad también muy alta, mientras que otras muestran regularmente unos niveles inferiores en ambas variables. Esta diferencia tenía implicaciones muy concretas, por lo que a la conducta demográfica se refiere. La clave identificativa de un régimen demográfico de alta o baja «presión» reside, en última instancia, en las prácticas nupciales. Hace algunos años, John Hajnal (1965) advirtió la existencia de dos modelos nupciales en Europa: uno, de matrimonio relativamente precoz y prácticamente universal, que predominaba en la Europa del Este y en muchas partes de la franja meridional del continente; y otro modelo de matrimonio tardío y restringido, característico del noroeste europeo. Aunque el matrimonio precoz y universal no se opone siempre de manera uniforme a una nupcialidad diferida y limitada (pues hay, por ejemplo, zonas de matrimonio relativamente temprano y, a la vez, relativamente restringido), las diferencias entre ambos modelos se advierten con suma claridad. En el siglo xvirI, la edad de las mujeres en sus primeras nupcias era cercana o superior a los 26 años en Bélgica, Inglaterra, Francia, Alemania y Escandinavia; mientras que en España, y probablemente en Italia, se situaba en torno a los 23 años (Flinn, 1981: 84). Los mapas existentes que muestran los valores de $I_{m}$ (véase apéndice) a finales del siglo xIX siguen repitiendo los mismos contrastes: en la Europa del Este y del Sur la nupcialidad era significativamente más intensa que en las restantes zonas del continente (Coale, 1969). Más allá de la correlación negativa que al parecer existe entre el volumen global de la nupcialidad y los niveles de fecundidad legítima, que diversos autores han advertido en muchas zonas (excepto en Inglaterra), los efectos sobre la descendencia legítima final tendrían que ser muy claros (Knodel, 1984; Van de Walle, 1978: 276-277; Wrigley y Schofield, 1983: 169-171). En la Europa meridional y centro-oriental esta elevada fecundidad quedaba reflejada en las tasas de natalidad, que tendian a situarse cerca o por encima del 40 por 1.000 , mientras que en otros sitios eran considerablemente inferiores. A su vez, la mortalidad era siempre superior en un régimen de «alta presión»: la esperanza de vida al nacimiento $\left(e_{0}\right)$ parece haber oscilado en la Europa meridional entre los 25 y los 28 años con anterioridad al siglo xIx, cuando más al norte de Europa solía şer de más de 30 , y frecuentemente cercana a los 40 años. Tales diferencias conciernen a todos los grupos de edades, aunque eran claramente más acusadas en las edades inferiores: en la Europa mediterránea la mortalidad infantil durante 
el primer año de vida $\left(q_{0}\right)$ normalmente superaba el 250 por 1.000 , mientras que en el Norte sus valores podían variar considerablemente, pero oscilando por lo común entre el 150 y el 225 por 1.000. Los contrastes de la mortalidad entre el primer aniversario y el quinto $\left({ }_{4} q_{1}\right)$ eran, sin embargo, todavía más sorprendentes: en las regiones europeas de «alta presión» solía ser casi tan elevada como $1 q_{0}$ y superaba normalmente el 200 por 1.000 , pero en las zonas de «baja presión» no pasaba, por regla general, del 100 por 1.000 .

Parece razonable pensar que, a fin de cuentas, un sistema demográfico de «alta presión» debe ser necesariamente una respuesta a los niveles de mortalidad imperantes. En un régimen de alta mortalidad la probabilidad de que un hijo sobreviva a su padre es relativamente baja, y si este último desea asegurar su propia sucesión debe tener cierto número de hijos (Wrigley, 1978). De acuerdo con las tablas-tipo de supervivencia, podemos estimar que una población donde $e_{0}=25$ años, necesitará una Tasa Bruta de Reproducción (TBR) (véase apéndice) de aproximadamente 2,7 hijas nacidas por mujer, simplemente para mantener su mismo tamaño en la generación siguiente, pero si la esperanza de vida se eleva a 35 años necesitará una TBR de sólo 1,9 , esto es, un 30 por 100 inferior (Coale y Demeny, 1966). Además, como la mortalidad adulta sería también más elevada (un 30-40 por 100 de diferencia en todos los grupos de edades entre ambos sistemas), el establecimiento de nuevas familias se vería más facilitado en el sistema de «alta presión» que en el de baja. La misma lógica sería válida también para el sexo femenino, puesto que las variaciones de la edad de acceso al matrimonio por parte de las mujeres debían de responder tanto a los niveles habituales de la mortalidad como a los de su fertilidad y su fecundidad natural (Mosk, 1981: 202204).

Una vez puesto en funcionamiento un determinado sistema matrimonial, todo un conjunto de condicionamientos ecológicos y culturales le ayudarían a mantenerse en vigencia. Por una parte, la nupcialidad era con toda seguridad la variable demográfica más sensible a los cambios económicos, y dado que matrimonio conducía más o menos directamente, en la mayor parte de Europa, a la formación de un hogar independiente, el acceso a la tierra, la disponibilidad de un trabajo y las circunstancias económicas en general actuaron como un filtro de la nupcialidad. Han sido muchos los historiadores que han sugerido que estas prácticas matrimoniales actuaron como mecanismo central a través del cual se impuso en la sociedad europea el «control preventivo» de Malthus (Wrigley y Schofield, 1981; Wrigley, 1983; Hajnal, 1982; Dupâquier, 1979; R. M. Smith, 1981a; Bideau, 1984). Pero, por otro lado, diversas fuerzas culturales pueden haber ayudado en muchas ocasiones a mantener en vigor ciertas prácticas nupciales, a pesar incluso de los cambios re- 
gistrados en las circunstancias económicas. La presión social que debían soportar al respecto las mujeres solteras en muchas zonas mediterráneas es un ejemplo concreto de ello. Motivaciones de este tipo pueden haber dificultado que el matrimonio oscilara de forma automática de acuerdo con la coyuntura económica, y por ello los cambios en la nupcialidad eran en muchos casos inapreciables, muy lentos, o se manifestaban con un visible retraso.

¿Hay evidencias concretas de que la nupcialidad se ajustaba a largo plazo a los niveles habituales de la mortalidad? Esta hipótesis ha originado algunas diferencias de opinión entre los historiadores, aunque en todo caso hay que admitir necesariamente una estrecha conexión entre ambas variables (Livi Bacci, 1978: 76-77; R. M. Smith, 1981b: 109-110; 1983: 124; Mosk, 1981). Sin adentrarnos en los aspectos obviamente mecanicistas de la hipótesis, parece claro que sistemas de nupcialidad y fecundidad intensas sólo se manifestaron en zonas caracterizadas por una elevada mortalidad. Además, aun cuando los niveles generales de fecundidad pudieran haber respondido a la mortalidad imperante, o incluso haber sido causados indirectamente por ella, esa misma fecundidad elevada conduciría, a su vez, a reforzar la mortalidad. En otras palabras, es razonable suponer que una elevada fecundidad acentuaría la mortalidad infantil y juvenil, lo que resulta claro si se piensa que los regímenes de «alta presión» conducían invariablemente a niveles de vida inferiores y a tasas de dependencia juvenil más altas (Wrigley, 1983; Watkins y Van de Walle, 1983).

¿Desde cuándo han existido estos diferentes sistemas demográficos? Según Hajnal, el «modelo matrimonial europeo» (esto es, el que aquí incluimos como propio del sistema de «baja presión») fue una creación bastante tardía y sólo se implantó con claridad en la Europa noroccidental hacia el siglo xvil (Hajnal, 1965: 116-120). Implícitamente, tanto él como M. Livi Bacci (1978: 76-77), sostienen que tras la Peste Negra el matrimonio juvenil y universal habría sido la norma, y que sólo mucho más tarde pasó a adoptarse de forma inequívoca el modelo nupcial europeo, en parte como respuesta al declive que empezaba a registrar la mortalidad. Existen, sin embargo, pruebas contradictorias acerca de la cuestión, al menos por lo que se refiere a los orígenes medievales de dicho modelo matrimonial. En primer lugar, Wrigley y Schofield $(1981: 230,424)$ han probado que, a mediados del siglo XvI, Inglaterra se hallaba ya claramente inmersa en un sistema de «baja presión». Richard Smith va más lejos en una serie de artículos recientes (1979: 81-85; 1981b: 114-117; 1983: 109-119), donde expresa sus dudas acerca de la interpretación tradicional de los registros de la capacitación inglesa de 1377 —pieza clave de la hipótesis de Hajnal-, y aporta poderosas razones para suponer que la edad de acceso al matrimonio ya era probablemente alta en la Inglaterra in- 
mediatamente posterior a la Peste Negra, y posiblemente también antes de 1349. Los cálculos de la esperanza de vida de los terratenientes ingleses efectuados por Russell (1979: 50), que la sitúan en 32 años en 1300-25 y en cerca de 33 en 1425-50, tienden a confirmar los argumentos de Smith. Un rápido cálculo nos muestra que con una esperanza de vida $\left(e_{0}\right)$ de 32,5 años y una TBR de 3,0 (típica de las zonas de «alta presión» demográfica), el crecimiento vegetativo $(r)$ se sitúa cerca del 1,4 por 100 al año, tasa que sólo llega a alcanzarse en Inglaterra en la primerasmitad del siglo xIx. En otras palabras, hay pruebas indirectas que parecen confirmar la existencia de un régimen de «baja presión» demográfica en la Inglaterra medieval. Recientes estudios locales han mostrado, por lo demás, que en algunas regiones inglesas, como Lincolnshire, hombres y mujeres se casaban relativamente tarde antes de la Peste Negra, y que el modelo occidental de nupcialidad tardía y limitada ya estaba bien establecido allí a mediados del siglo xIv (Hallam, 1985).

Por otra parte, la situación era muy diferente en la Europa mediterránea. La información disponible sobre la edad en las primeras nupcias en la Italia de la segunda mitad del siglo xIv y del siglo xv en el sur de Francia, antes y después de la Peste Negra, y en la España del siglo xvi indica el predominio del matrimonio precoz en todas estas zonas (Herlihy y Klapish-Zuber, 1978: 204-209; Le Roy Ladurie, 1975: 275-279; Valero, 1984: 42-46), donde, además, la esperanza de vida $\left(e_{o}\right)$ oscilaba, en pleno siglo xvirI, entre los 25 y los 28 años (Del Panta y Livi Bacci, 1980: 105; Livi Bacci, 1968: $90,92)$. Todos estos datos nos inducen a pensar que, inmediatamente después de la Peste Negra, distintas regiones de Europa ya se encontraban probablemente caracterizadas por los diferentes sistemas demográficos que iban a perdurar allí hasta los tiempos modernos.

Por debajo de estos sistemas demográficos, definidos sobre todo por el modelo de nupcialidad propio de cada uno, la experiencia europea registraba, al parecer, niveles muy variables de mortalidad no catastrófica u «ordinaria». En sucesivos momentos, entre 1200 y 1850 , pueden encontrarse diferencias de $e_{\mathrm{o}}$ hasta 15 años. Aunque estos valores de la esperanza de vida también varían a lo largo de los siglos en el interior de ciertas regiones, los niveles de la mortalidad del norte o del sur de Europa se mueven dentro de bandas claramente separadas: en el Norte, la $e_{o}$ oscilaba entre los 30 y los 40 años, mientras que en el Sur y en el Este nunca alcanzó los 30 hasta finales del siglo xvill, en algún caso, o, por lo general, hasta bien entrado el siglo XIX. $\mathrm{Ya}_{\mathrm{a}}$ hemos sugerido que los niveles de mortalidad imperantes parecen estar en el origen de los sistemas de alta y baja «presión», por lo que el tema de los contrastes de mortalidad en uno y otro régimen demográfico requiere un análisis más detenido. Una importante consideración al respecto ha de insistir 
en la persistencia a lo largo del tiempo de estos contrastes, lo que tàl vez indica que la mortalidad secular respondía dentro de cada región a factores que tenían poco que ver con variables económicas coyunturales, como los salarios o los precios, aunque reflejara notables diferencias económicas de «larga duración» entre unas regiones y otras. La primera de estas hipótesis ha sido verificada al correlacionar mortalidad y precios durante periodos relativamente breves, aunque parece igualmente plausible si se aplica a períodos más extensos (R. Lee, 1973; 1978: 170-186). Existe, sin embargo, una conexión muy concreta entre las diferencias climáticas y las que presentaba la mortalidad. $R$. Lee ha abordado este problema con referencia a todos los grupos de edades y ha mostrado la existencia de una relación muy estrecha entre temperatura y mortalidad, pero un enfoque comparativo norte-sur aconsejaría limitarse a la relación entre las condiciones climáticas y la mortalidad infantil y juvenil (Lee, en Wrigley y Schofield, 1981: cap. 9; Galloway, 1984). La cantidad de niños que desaparecían víctimas de enfermedades propias de la estación estival, a causa sobre todo de trastornos digestivos frecuentemente relacionados con la dentición (que solía coincidir con el final de una prolongada lactancia materna), influía poderosamente en los modelos estacionales de la mortalidad en el sur de Europa. Los datos, agregados a escala nacional, muestran que mientras los máximos de invierno y de comienzos de la primavera son típicos, con ligeras diferencias, de la curva estacional de la mortalidad en Inglaterra, Escocia, Finlandia, Holanda, Francia y el norte de Alemania, los meses de verano destacan claramente como los de máxima mortalidad en la Europa mediterránea hasta comienzos del siglo xx (Wrigley y Schofield, 1981: 297; Ferrari y Livi Bacci, 1985). Tales contrastes se explican casi del todo por las diferencias en la mortalidad infantil y juvenil, donde el grupo de 1.4 años tiene un peso específico muy relevante.

Junto a estas variaciones relacionadas de alguna forma con las diferencias climáticas, otros'factores contribuían también a mantener los contrastes de la mortalidad tan evidentes entre las distintas regiones europeas. Los regímenes de «alta presión» parecen conducir invariablemente, como ya se dijo, a niveles de vida inferiores, pero no sólo por tener que soportar mayores proporciones de población dependiente de edades jóvenes, sino probablemente también a causa de la mayor inflexibilidad de la nupcialidad y la fecundidad para reaccionar en tales sistemas ante el cambio económico. El cuidado de los menores se vería altamente dificultado $\mathrm{y}$, por otro lado, la alimentación infantil sería con toda seguridad más precaria en esas zonas de alta fecundidad. La conexión entre nutrición y mortalidad es aún una cuestión abierta que sigue provocando una gran división de opiniones entre los especialistas (Livi Bacci, 1983; Drake, 1979: 292-294; Fridlizius, 1979: 349-350; W. R. Lee, 1979: 
15-17). La información actual procedente de los países en vías de desarrollo indica, sin embargo, que los niveles de nutrición, cuando descienden por debajo de un determinado punto, pueden llegar a debilitar considerablemente la resistencia frente a infecciones de origen exógeno de la población de menos de 5 años de edad, que era precisamente la que acusaba una mayor mortalidad en el mundo mediterráneo (Watkins y Van de Walle, 1983: 219-224). Aunque no existen pruebas concluyentes sobre esta cuestión, la hipótesis es atractiva y ayudaría a explicar los mecanismos por los cuales una alta fecundidad acabaría siendo no sólo el resultado, sino también la causa de unos elevados niveles de mortalidad.

La existencia de diferentes sistemas demográficos tiene numerosas implicaciones para el movimiento de la población, que parece conveniente señalar. En primer lugar, la influencia de la mortalidad sobre las tasas de crecimiento es, por lo común, mucho mayor en un sistema de «alta presión» que en otro de «baja presión» (Wrigley y Schofield, 1981: 236-248). Buena prueba de ello es que una población donde, caeteris paribus, al menos la mitad de las defunciones corresponden a los menores de 7 años (situación típica de la Europa mediterránea), y donde la tasa bruta de natalidad sea sólo un 10 por 100 superior a la de mortalidad, una reducción del 20 por 100 en los niveles de esa mortalidad infantil y juvenil bastará por sí sola para duplicar las tasas de crecimiento natural (Pérez Moreda, 1980: 146). En segundo lugar, en los sistemas de «alta presión» la nupcialidad, y en consecuencia la fecundidad, tienen escasas posibilidades de actuar generando un decisivo aumento de las tasas de crecimiento a largo plazo (exceptuamos, claro está, las bruscas variaciones en el calendario de la nupcialidad y el incremento de las segundas nupcias que solían producirse durante algunas crisis agudas de mortalidad, y sobre todo inmediatamente después de las mismas). Esto era así precisamente porque ya alcanzaban por lo común niveles próximos a los que permitía su máxima capacidad posible. La única oportunidad realista de un cambio relevante en este sentido consistiría en un incremento de la fecundidad legítima: algo que, aunque teóricamente posible, no parece que haya tenido lugar en un grado significativo durante el período analizado. Por otra parte, en los sistemas de «baja presión», la nupcialidad podía adaptarse mejor y más ágilmente al cambio económico, estimulando el crecimiento demográfico mediante una progresiva intensificación. Curiosamente, pocas regiones europeas conocieron una intensificación a largo plazo de su nupcialidad; pero allí donde esto ocurrió fue en respuesta de unas condiciones económicas más favorables, como en Inglaterra, o donde el incremento de la fecundidad total mediante este mecanismo estaba siendo anulado pòr un declive simultáneo de la fecundidad legítima, como es el caso francés (Henry y Houdaille, 1978: 50-51, 
57-58; 1979: 413). En otros países de «baja presión», concretamente en Escandinavia, los aumentos temporales registrados por la nupcialidad a escala nacional sólo tuvieron efectos pasajeros, aunque positivos, sobre el crecimiento demográfico. La doble contribución al crecimiento a través del incremento de la fecundidad y del declive de la mortalidad condujo en las regiones de «baja presión» a tasas de crecimiento bastante superiores a las de las zonas mediterráneas, donde los niveles de fecundidad tendían a reducirse progresivamente con objeto de compensar el gradual descenso de la mortalidad.

Otra implicación de los diferentes sistemas de presión demográfica afectaría, en este caso, a las distintas modalidades de crecimiento económico que se registran en Europa durante el período de la primera industrialización. Por una parte, el equilibrio entre población y recursos se alcanzaría en las áreas de «alta presión» en un punto próximo al nivel de subsistencia, con el consiguiente perjuicio para las posibilidades de ahorro en dichas zonas, que serían precisamente las que más iban a tardar en incorporarse al proceso de modernización económica (Wrigley y Schofield, 1981: 459-461). Por otro lado, con una fecundidad y mortalidad elevadas, la proporción de población en los grupos de edades inferiores sería siempre claramente mayor que en las zonas de fecundidad y mortalidad reducidas (hasta un 3-4 por 100 más numeroso el grupo de $0-14$ años con $e_{o}=25$ que con $e_{o}=35$ años), reduciendo así no sólo la proporción de mano de obra potencial, sino comprometiendo también el mismo crecimiento económico a largo plazo (Spengler, 1972).

Resulta de interés mostrar la interacción de estas variables en Europa entre 1750 y 1850 , un período en que la mortalidad catastrófica había empezado a declinar considerablemente, pero anterior aún al desarrollo de la llamada "transición demográfica" en la mayoría de los países. No necesitamos subrayar la importancia que confiere a este período la Primera Revolución Industrial, y pasaremos a centrar nuestra atención sobre las tasas de crecimiento demográfico, en aumento por todas partes, aunque en grado muy variable. En este sentido, las regiones europeas observadas aparecen divididas entre estas fechas en cuatro grupos bien diferenciados: Inglaterra, cuya población casi se triplica; Alemania, los Países Bajos y Escandinavia, cuyas poblaciones se multiplican por dos, aproximadamente; Italia y España, cuyos efectivos demográficos crecen un $60-65$ por 100 , y, finalmente, Francia, donde sólo aumentan' un 50 por 100 .

El caso de Inglaterra $-y$, con algunas importantes excepciones, de las Islas Británicas - es bien conocido y constituye un buen ejemplo de la importancia de la nupcialidad como estímulo del crecimiento demográfico. Durante la primera mitad del siglo xviII la nupcialidad y la fecundidad crecieron apoyadas en el alza de los salarios. Aunque los niveles de vida tendieron a 
caer en la segunda mitad de la centuria, bajo la presión de un inusitado crecimiento demográfico, la reacción de ambas variables demográficas se retrasó manteniendo el mismo ritmo de crecimiento hasta un momento en que la dependencia de la población respecto a la producción agrícola había sido eliminada por la incipiente revolución industrial a comienzos del siglo XIX (Wrigley y Schofield, 1981: 402-453; Wrigley, 1983). De esta forma, nunca descendió realmente la tasa de crecimiento demográfico a lo largo del período. Aunque el declive de la mortalidad desempeñó un papel importante en tal proceso, su peso relativo habría sido menor que el de la nupcialidad, que, sin un incremento visible de la fecundidad legítima, y en ausencia de un masivo control de la fecundidad, era él único factor que determinaba las oscilaciones de la fecundidad total (Wrigley y Schofield, 1983: 169-170; Wilson, 1984).

El segundo grupo de países presenta un tipo de comportamiento más heterogéneo, aunque - al menos en dos de ellos- la fecundidad parece haber ayudado de forma importante, aunque fugaz, a elevar las tasas de crecimiento natural. En los Países Bajos conviene distinguir entre Bélgica, que conoció un fuerte crecimiento a lo largo de todo el período, y Holanda, que sólo empieza a crecer de forma sostenida a comienzos del siglo XIX. Por la información disponible sobre Bélgica, algunas zonas parecen haber registrado allí un incremento de la nupcialidad durante el siglo xviII, y posiblemente también un aumento paralelo de la fecundidad legítima, aunque aquí las opiniones no son totalmente coincidentes (Deprez, 1965: 615, 620; 1979: 271-272; Vandenbroeke, 1984; Mendels, 1984). Los niveles más altos de fecundidad legítima y general se alcanzan, tanto en Holanda como en Bélgica, entre 1825 y 1835, para empezar a descender después en esa última fecha. La nupcialidad experimentó serias restricciones después de las primeras décadas del siglo, posiblemente debidas a los efectos demográficos de la desaparición de la industria rural. Por otra parte, la mortalidad general, que había permanecido estacionaria hasta mediados del siglo xviII, empezó a caer desde 1780 y perdió entre un 12 y un 20 por 100 de sus niveles previos. Esta caída cesó, sin embargo, a partir de 1830 , cuando se advierte incluso una ligera elevación de la mortalidad infantil, que ha sido atribuida a cambios en las prácticas tradicionales de lactancia (Vandenbroeke et al., 1983: 260). En Escandinavia parece haber tenido lugar una combinación parecida de declive de la mortalidad e incremento, temporal al menos, de la fecundidad. Pero a diferencia de los Países Bajos, donde no está claro cuál de las dos variables fue la predominante, en Suecia parece que fueron las mejoras registradas por la mortalidad el factor clave del crecimiento, sólo momentáneamente ayudado, entre 1800 y 1830, por algún aumento de la nupcialidad y de la fecundidad general (Drake, 1979; Fridlizius, 1979; Hofsten y Lundström, 1976). La caída 
de la mortalidad afectó más a la población infantil que a los adultos, y ha sido atribuida a mejoras en la nutrición relacionadas con la introducción masiva de la patata poco después de 1800 . En Alemania, ni la fecundidad ni la mortalidad mostraron cambios significativos antes de 1850 (Knodel, 1986; W. R. Lee, 1979). Algunos han sugerido la posibilidad de un incipiente control voluntario de la fecundidad, que acabaría siendo encubierto por una creciente fertilidad, hipótesis ésta bien peculiar y fascinante, que habría dado como resultado unos niveles invariables de fecundidad total (Knodel, 1986; Knodel y Wilson, 1981). La clave explicativa del crecimiento demográfico alemán residió seguramente en sus tasas de crecimiento natural, que, en ausencia de mortalidad catastrófica, reflejaban la importancia de unos altos niveles de fecundidad legítima ( $I_{g}$ entre 0,77 y 0,80 a lo largo de todo el período) (véase apéndice). Pero la verdadera aceleración de la población alemana se registró después de 1850 y fue posible gracias a una fuerte caída de la mortalidad general (W. R. Lee, 1979: 195).

Las poblaciones de Italia y España no sólo aumentaron a un ritmo más lento - sin que en ello influyeran las pérdidas, poco apreciables, debidas a la emigración-, sino que también revelan un modelo de crecimiento demográfico claramente distinto del de sus vecinos del norte de Europa. Hay razones para creer que en ambos países la mortalidad general y la fecundidad legítima descendieron ligeramente durante el período en cuestión (Livi Bacci, 1968: 90, 92, 97; Bellettini, 1980: 40; Bellettini y Samoggia, 1982: 16; Del Panta y Livi Bacci, 1980: 103, 118). Este incipiente declive de la fecundidad ha sido atribuido a un control voluntario entre ciertos sectores sociales en un intento de compensar la caída de la mortalidad y como alternativa a una emigración que en este período había cesado casi por completo (Livi Bacci, 1977: 47-48). El descenso de la mortalidad dio paso también a una restricción progresiva de la nupcialidad, que se pone de manifiesto - más en España que en Italia - en la elevación de la edad de acceso al matrimonio, parcialmente compensada en el caso éspañol por el declive del celibato definitivo (Bellettini, 1980: 40; Del Panta y Livi Bacci, 1980: 126-127; Pérez Moreda, 1985). Parece claro que en ambos países una moderada caída de la mortalidad pasó a ser compensada por medio de restricciones en la fecundidad y la nupcialidad, con el resultado final de un crecimiento más modesto que en los ejemplos anteriores, pero sostenido, sobre todo a partir de 1815 .

Francia fue realmente, como han señalado algunos historiadores, un caso «único en Europa» durante más de medio siglo (Van de Walle, 1979; Wrigley, 1985). Su crecimiento demográfico, a partir de 1750 , fue con diferencia el más bajo de toda Europá debido a la combinación de una mortalidad relativamente elevada y una fecundidad en franco declive. En este caso 
la mortalidad llevó claramente la iniciativa del proceso, acusando profundos cambios desde la segunda mitad del siglo xviII. Entre 1750 y 1770 , el indicador $e_{o}$ se situaba en Francia en torno a los 29 años, cuando en Inglaterra era ya de 35,7, pero un siglo más tarde la esperanza de vida era casi idéntica en ambos países: en Francia había aumentado un 41 por 100 y sólo un 13 por 100 en Inglaterra (Blayo, 1975: 141; Van de Walle, 1979: 142). La mortalidad infantil siguió la misma senda cayendo casi un 30 por 100 entre 1789 y 1819. En un primer momento la nupcialidad reaccionó "negativamente para equilibrar los efectos del declive de la mortalidad: la edad de acceso al matrimonio se fue elevando hasta 1789 y los niveles de soltería definitiva hicieron otro tanto. Pero cuando el control voluntario de la fecundidad empezó a manifestarse masivamente, la nupcialidad se vio relevada, por así decir, de su responsabilidad en el recorte de la fecundidad y se recuperó de forma notable a lo largo del siglo xix (Henry y Houdaille, 1978: 50-51, 57-58; 1979: 413, 420). En este sentido, el comportamiento de la nupcialidad francesa fue único en Europa durante la primera mitad del ochocientos. La fecundidad legítima también conoció un declive continuo desde 1740, aunque esta tendencia sólo cobró verdadera importancia tras la Revolución Francesa. En 60 años la fecundidad legítima descendió casi un 24 por 100, y de 1770-1789 a $1790-1819$ perdió entre un 12 y un 18 por 100 , según las regiones. El proceso continuó a lo largo de la primera mitad del siglo xIx (Henry, 1978: 886; Houdaille, 1976: 353; Henry, 1972: 979; Henry y Houdaille, 1973: 889). A mediados de esta centųria los valores de $I_{g}$ eran en Francia bastante inferiores a los de cualquier otro país europeo (Van de Walle, 1979: 143). La nupcialidad nunca reaccionó como en Inglaterra, porque la Francia del siglo xviri no mostró signo alguno de mejores condiciones de vida. Pero la rápida caída de la mortalidad anuló las capacidades de la nupcialidad para limitar el crecimiento de la población. Es posible que las mejoras registradas en la mortalidad, junto al deseo del campesino francés de preservar sus niveles de vida, le condujeran a cambiar radicalmente su apreciación del valor de cada nacimiento y a practicar métodos de control de la fecundidad que ya eran conocidos y aplicados desde la segunda mitad del siglo xviII. Este cambio de actitud se vio probablemente facilitado por las implicaciones culturales $\mathrm{y}$ la normativa jurídica derivadas del proceso revolucionario, y tendió a manifestarse antes y de forma más intensa precisamente en las regiones caracterizadas por una fecundidad más elevada: el nordeste y el sudeste de Francia (Berkner y Mendels, 1978). En cualquier caso, la especailidad de la experiencia francesa resulta patente, aunque sus razones últimas probablemente seguirán siendo objeto de investigación y debate durante algún tiempo, como lo fueron ya en la época de Le Play. 
La caída secular de la fecundidad junto al descenso, igualmente importante, de la mortalidad no tardarían en afectar finalmente a toda Europa, dando origen a lo que se conoce como la «transición demográfica», cuya consecuencia lógica ha sido una progresiva uniformidad de las tasas de crecimiento en el continente. Podríamos considerar, como ha hecho algún historiador observando a la luz del presente los últimos tres siglos, que todo el proceso fue poco más que un vacilante ajuste en pos del modelo de comportamiento que iba a generalizarse en nuestro siglo (McKeown, 1976). Pero ¿por qué contentarnos con tal simplificación? Lo maravilloso de la Historia reside en su diversidad, y precisamente la investigación de la diversidad de comportamientos demográficos, tanto nacionales como regionales, debería ser un objetivo primordial de los historiadores de la población en los años venideros.

\section{APENDICE}

Los índices $I_{m}$ e $I_{g}$ fueron elaborados por Ansley J. Coale en el marco del European Fertility Project de la Universidad de Princeton (Coale, 1965; 1969). En ambos índices, los niveles respectivos de nupcialidad y fecundidad se ponen en relación con la fecundidad de las mujeres hutteritas entre 1921 y 1930. Esta fecundidad es una de las más altas que se conocen en poblacoines históricas, y se aproxima a lo que podríamos considerar la fecundidad máxima posible en ausencia de cualquier tipo de impedimento social, económico o cultural que pudiera limitarla. La fecundidad de las hutteritas en los distintos grupos de edades era: 0,300 (15-19), 0,550 (20-24), 0,502 (25-29), 0,447 (30-34), 0,406 (35-39), 0,222 (40-44) y 0,061 (45-49) (Henry, 1961).

$I_{m}$ se puede expresar de la siguiente manera:

$$
I_{m}=\frac{\Sigma c_{i} \cdot F_{i}}{\Sigma m_{i} \cdot F_{i}}
$$

donde $c_{i}$ son las mujeres casadas a edad $i ; m_{i}$ son las mujeres de edad $i, \mathrm{y}$ $F_{i}$ es la fecundidad de las hutteritas de edad $i$. El uso de la fecundidad de las hutteritas como ponderación de un índice de nupcialidad implica primar las edades en las que las mujeres tienen una fecundidad mayor, lo que supone una estandarización por edades de la población femenina. 
$I_{g}$ se puede expresar así:

$$
I_{g}=\frac{N_{l}}{\sum c_{i} \cdot F_{i}}
$$

donde $N_{l}$ son los nacimientos legítimos; $c_{i}$ son las mujeres casadas a edad $i$, y $F_{i}$ es la fecundidad de las hutteritas de edad $i$. Es decir, dicho índice mide la fecundidad legítima relativa de una población determinada, que alcanzaría un valor de 1 si fuese igual a la de las hutteritas.

Finalmente, la Tasa Bruta de Reproducción (TBR) es el equivalente al número de hijas nacidas a una cohorte hipotética de mujeres con unas tasas específicas de fecundidad determinadas y en ausencia de mortalidad.

\section{BIBLIOGRAFIA}

APPLEBY, A. B. (1980): «The disappearance of plague: A continuing puzzle», The Economic History Review, 33, 2: 171-173.

Bellettini, A. (1980): «L'evoluzione demografica dell'Italia nel quadro europeo del Settecento: analogie e particolarità", en S.I.DE.S. (ed.): La popolazione italiana nell Settecento, Bolonia: 13-70.

BelletTINI, A., y SAM OGGIA, A. (1982): «Premières recherches sur les tendances de longue période de la mortalité infantile dans la campagne de Bologne (XVII'-XIX ${ }^{e}$ siècles)», Genus, vol. 37, núms. 1-2: 1-26.

BERKNER, L. K., y MENDELS, F. F. (1978): «Inheritance systems, family structure, and demographic patterns in western Europe, 1700-1900», en TILLY, Ch. (ed.): Historical Studies in Changing Fertility, Princeton: 209-223.

BideAu, A. (1984): «Les mécanismes autorégulateurs des populations traditionnelles», $A n$ nales ESC, 39: 1040-1057.

Biraben, J.-N. (1975-1976): Les bommes et la peste en France et dans les pays européens et méditerranéens, 2 vols., París y La Haya.

Blayo, Y. (1275): «La mortalité en France de 1740 à 1829», Population, 30: 123-142.

COALE, A. J. (1965): «Factors associated with the development of low fertility: An historic summary», en Naciones. Unidas: World Population Conference, 2: 195-209.

- (1969): «The decline of fertility in Europe from the French Revolution to World War II», en Ber hrman, S. J.; CoRSA, L., y FreEdman, R. (eds.): Fertility and Family Planning: $A$ World View, Michigan: 3.24.

Conle, A. J., y Demeny, P. (1966): Regional Model Life Tables and Stable Populations, Princeton.

Del Panta, L. (1978): «Italy», en LEE, W. R. (ed.): European Demograpby and Economic Growth, Londres: 196-234.

- (1980): Le epidemie nella storia demografica italiana (secoli XIV-XIX), Turín.

Del Panta, L., y Livi BaCcI, M. (1977): "Chronologie, intensité et diffusion des crises de mortalité en Italie: 1600-1850», Population, 32 (número especial): 401-440.

- (1980): «Le componenti naturali dell'evoluzione demografica nell'Italia del Settecentom, en S.I.DE.S. (ed.): La popolazione italiana nell Settecento, Bolonia: 71-139. 
DePrez, P. (1965): *The demographic development of Flanders in the eighteenth century», en Glass, D. V., y Eversley, D. E. C. (eds.): Population in History. Essays in Historical Demography, Londres: 608-630.

- (1979): "The Low Countries", en LEE, W. R. (ed.): European Demography and Economic Growth, Londres: 236-283.

De VRIES, J. (1974): The Dutch Rural Economy in the Golden Age, 1500-1700, New Haven y Londres.

Drake, M. (1979): «Norway», en LeE, W. R. (ed.): European Demography and Economic Growth, Londres: $284-318$.

Dupaquier, J. (1972): «De l'animal à l'homme: le mécanisme autorégulateur des populations traditionnelles», Revue de l'Institut de Sociologie, 2: 177-211.

- (1979): La population rurale du Bassin Parisien à l'époque de Louis XIV, París-Lille.

Ferrari, G., y Livi BAcCI, M. (1985): «Sulle relazioni tra temperatura e mortalità nell'Italia unita, 1861-1914\%, L'evoluzione demografica dell'Italia nel secolo XIX, Bolonia: 273-298.

FLINN, M. W. (1974): «The stabilization of mortality in pre-industrial western Elurope», Journal of Economic History, 3: 285-318.

- (1979): «Plague in Europe and the Mediterranean Countries», Journal of European Economic History, 8, 1: 131.148.

- (1981): The European Demographic System, Baltimore.

FridliziUs, G. (1979): «Sweden», en LEE, W. R. (ed.): European Demography and Economic Growth, Londres: $340-405$.

Goubert, P. (1968): Cent mille provinciaux au XVII' siecle, París.

Hallam, H. E. (1985): «Age at first marriage and age at death in the Lincolnshire Fenland, 1252-1478", Population Studies, 39, 1: 55-69.

Hajnal, J. (1965): «European marriage patterns in perspective», en Glass, D. V., y Eversley, D. E. C.: Population in History. Essays in Historical Demography, Londres: 101-146.

- (1982): «Two kinds of preindustrial household formation system*, Population and Development Review, 8, núm. 3: 449-494.

HenRy, L. (1961): «Some data on natural fertility», Eugenics Quarterly, 8: 81-91, esp. 84.

- (1972): «Fécondité des mariages dans le quart sud-ouest de la France de 1720 à 1829» (suite), Annales ESC, vol. 27: 977-1023.

- (1978): «Fécondité des mariages dans le quart sud-est de la France de 1670 a 1829», Population, 33.

HenRY, L., y Houdaille, J. (1973): «Fécondité des mariages dans le quart nord-ouest de la France de 1670 à 1829», Population, 28: 873-924.

- (1978): «Célibat et age au mariage aux XVIII ${ }^{*}$ et XIX $^{e}$ siécles en France. I. Célibat définitif», Population, 33: 43-84.

- (1979): "Célibat et age au mariage aux $x^{2} I^{*}$ et $x^{*}$ siècles en France. II. Age au premier mariage», Population, 33: 403-442.

Herligy, D., y KLAPISC h-Zuber, C. (1978): Les Toscans et leurs familles: Une étude du catasto florentin de 1427, París.

HofsTEN, E., y LUNDSTRöm, H. (1976): Swedish population bistory. Main trends from 1750 to 1970 , Estocolmo.

Houdaille, J. (1976): «La fécondité des mariages de 1670 à 1829 dans le quart nord-est de la France», Annales de Démographie Historique: 341-391.

- (1984): *La mortalité des enfants dans la France rurale de 1690 à 1779», Population, 39: $77-106$.

KNODEL, J. (1986): «Demographic transitions in German villages», en CoALE, A., y WatkINS, S. (eds.): The Decline of Fertility in Europe, Princeton: 337-389.

KNODEL, J., y WILSON, C. (1981): *The secular increase in fecundity in German village populations: An analysis of reproductive histories of couples married 1750-1799», Population Studies, 35: 53-84. 
Lebrun, F. (1980): «Les crises démographiques en France aux XvII et XvIII siècles», $A n$ nales ESC, 35, 2: 205-234.

Le Roy Ladurie, E. (1975): Montaillou, village occitan de 1294 à 1324, París.

LEE, R. D. (1973): «Population in pre-industrial England: en econometric analysis», Quarterly Journal of Economics, 87: 581-607.

- (1978): "Models of pre-industrial population dynamics, with applications to England», en Tilly, C. (ed.): Historical Studies in Changing Fertility, Princeton.

LEE, W. R. (1979): European Demography and Economic Growth, Londres.

Livi BACCI, M. (1968): «Fertility and nuptiality changes in Spain from the late 18th to the early 20th century», Population Studies, 22: 83-102, 211-234.

- (1977): A History of Italian Fertility during the Last Two Centuries, Princeton.

- (1978): La société italienne devant les crises de mortalité, Florencia.

- (1983): «The nutrition-mortality link in past times: A comment», Journal of Interdisciplinary History, 14, 2; 293-298.

McEvedy, C., y Jones, R. (1978): Atlas of World Population History, Londres.

MCKEOWN, T. (1976): The modern rise of population, Londres.

- (1978): «Fertility, mortality and causes of death. An examination of issues related to the modern rise of population», Population Studies, 32: 535-542.

MCNeIll, W. H. (1976): Plagues and peoples, Nueva York.

Mendels, F. (1984): "Niveau des salaires et âge au mariage en Flandre, Xvir'-xviIr ${ }^{\star}$ siècles», Annales ESC, 39, 5: 939.955.

Mosk, C. (1981): "The evolution of premodern demographic regimes: A research note», Explorations in Economic History, 18: 199-208.

NADAL, J. (1984): La población española (Siglos XVI a XX), Barcelona.

PÉrez MoredA, V. (1980): Las crisis de mortalidad en la España interior, siglos XVI-XIX,

- (1984): «Crisis demográficas y crisis agrarias: paludismo y agricultura en España a fines del siglo xvirI", Congreso de Historia Rural: 333-354.

- (1985): «La evolución demográfica española en el siglo xIx (1797-1930): tendencias generales y contrastes regionales», en S.I.DE.S. (ed.): La popolazione italiana nell'ottocento. Continuità e mutamenti, Bolonia: 45-113.

Russell, J. C. (1979): «La poblaciór en Europa del año 500 al 1500», en Cipolla, C. (ed.): Historia Económica de Europa (1). La Edad Media, Barcelona: 25-77.

SLACK, P. (1981): «The disappearance of plague: an alternative view», The Economic History Review, 24, 3: 469-476.

SMITH , R. M. (1978): «Population and its geography in England 1500-1700», en DoDGSH ON, R. A., y Butuin, R. A. (eds.): A bistorical geograpby of England and Wales, Londres y Nueva York.

- (1979): «Some reflections on the evidence for the origins of the "European marriage pattern" in England», en HARRIS, C. (ed.): The sociology of the family, Keele: 74-112.

- (1981 a): «Fertility, economy and household formation in England over three centuries», Population and Development Review, 7, 4: 595-622.

- (1981 b): «The people of Tuscany and their families in the fifteenth century: medieval or Mediterranean?», Journal of Family History, 6, 1: 107-128.

- (1983): wHypothèses sur la nuptialité en Angleterre aux XIII'-xIV siècles», Annales ESC, 1: 107-136.

SPENGLER, J. J. (1972): «Demographic factors and early modern economic development», en Glass, D. V., y Revelde, R. (eds.): Population and Social Change, Londres: 87-98.

VAlERo, A. (1984): "Edad media de acceso al matrimonio en España. Siglos XVI-XIX», Boletín de la Asociación de Demografía Histórica, 2, 2: 39.48.

VANDENBRoEKE, C. (1984): "Le cas flamand: évolution sociale et comportements démographiques aux XVII'-XIX ${ }^{c}$ siècles", Annales ESC, 39, 5: 915-938.

Vandenbroeke, C.; VAN Poppel, F., y Van DeR Woude, A. M. (1983): «Le développement séculaire de la mortalité aux jeunes âges dans le territoire du Benelux», Annales de Démographie Historique: 257-289. 
VAN DE WaLle, E. (1978): "Alone in Europe: the French fertility decline until 1850", en TIlly, C. (ed.): Historical Studies in Changing Fertility, Princeton: 257-288.

- (1979): «France», en LEE, W. R. (ed.): European Demography and Economic Growth, Londres: 123-143.

Watkins, S. C., y VAN dE WALle, E. (1983): «Nutrition, mortality and population size: Malthus' court of last resort», Journal of Interdisciplinary History, 14, 2: 205-226.

Wilson, C. (1984): «Natural fertility in pre-industrial England, 1600-1799», Population Studies, 38, 2: 225-240.

WRIGLEY, E. A. (1978): «Fertility strategy for the individual and the group», en TILly, C. (ed.): Historical Studies in Changing Fertility, Princeton: 135-154.

- (1983): "The growth of population in eighteenth-century England: A conundrum resolved", Past and Present, 98: 121-150.

- (1985): «The fall of marital fertility in nineteenth-century France: Exemplar or Exception?" (primera y segunda partes), European Journal of Population, 1: $31-60$ y 141-177.

WRIGLEY, E. A., y SCH OFIELD, R. S. (1981): The population history of England, 15411871; A reconstruction, Cambridge (Mass.).

- (1983): «English population history from family reconstitution: Summary results, 1600 . 1799», Population Studies, 37, 2: 157-184. 